\title{
miRNA-146a attenuates inflammation in an in vitro spinal cord injury model via inhibition of TLR4 signaling
}

\author{
YING TAN $^{1 *}$, LONGTAN YU $^{1 *}, \mathrm{CHUNMING} \mathrm{ZHANG}^{2,3}, \mathrm{KEBING} \mathrm{CHEN}^{4}, \mathrm{JUNFAN} \mathrm{LU}^{1}$ and LEI TAN ${ }^{1}$ \\ ${ }^{1}$ Department of Spine Surgery, Weifang Traditional Chinese Medicine Hospital, Weifang, Shandong 261041; \\ ${ }^{2}$ School of Medicine, Shandong University, Jinan, Shandong 250012; ${ }^{3}$ Department of Orthopedics, \\ Shandong Provincial Qianfoshan Hospital, Shandong University, Jinan, Shandong 250014; ${ }^{4}$ Department of Spine Surgery, \\ Third Affiliated Hospital of Southern Medical University, Guangzhou, Guangdong 510430, P.R. China
}

Received June 23, 2017; Accepted November 17, 2017

DOI: $10.3892 /$ etm.2018.6645

\begin{abstract}
The present study evaluated the anti-inflammatory effect of microRNA (miR)-146a in a spinal cord injury (SCI) rat model and in vitro model, and explored possible underlying mechanisms of this effect. miR-146a expression was analyzed using reverse transcription-quantitative polymerase chain reaction (RT-qPCR). Tumor necrosis factor (TNF)- $\alpha$, interleukin (IL)-1 $\beta$ and IL-6 content was measured using ELISA kits. Inducible nitric oxide synthase (iNOS), prostaglandin E2 (PGE2), Toll-like receptor 4 (TLR4), myeloid differentiation primary response 88 (MyD88) and phosphorylated (p)-nuclear factor $(\mathrm{NF})-\kappa \mathrm{B}$ were measured using western blotting. In the SCI rat model, miR-146a expression was downregulated. In the in vitro model, downregulation of miR-146a increased inflammation, enhanced iNOS and PGE2 protein expression and induced TLR4, MyD88 and NF- $\kappa \mathrm{B}$ expression. Overexpression of miR-146a reduced inflammation, iNOS and PGE2 protein expression, and suppressed TLR4, MyD88 and $\mathrm{NF}-\kappa \mathrm{B}$ expression in the in vitro $\mathrm{SCI}$ model. The inhibition of TLR4 attenuated the proinflammatory effects of anti-miR-146a in the in vitro SCI model. The results indicate that miR-146a reduces inflammation in an SCI model through the TLR4-NF- $\kappa \mathrm{B}$ signaling pathway. The present study demonstrated that miR-146a may be a promising therapeutic agent for SCI.
\end{abstract}

Correspondence to: Dr Chunming Zhang, Department of Orthopedics, Shandong Provincial Qianfoshan Hospital, Shandong University, 16766 Jingshi Road, Jinan, Shandong 250014, P.R. China E-mail: zhangsurgeon@qq.com

Dr Kebing Chen, Department of Spine Surgery, Third Affiliated Hospital of Southern Medical University, 183 Zhongshan Avenue (West), Guangzhou, Guangdong 510430, P.R. China

E-mail: chenkebing.sysu@gmail.com

*Contributed equally

Key words: microRNA-146a, inflammation, Toll-like receptor 4, nuclear factor $\kappa \mathrm{B}$, spinal cord injury

\section{Introduction}

Patients with spinal cord injury (SCI) suffer from extreme pain while exercising (1). This results in a notable decrease in quality of life, and a heavy burden on family members and society (2). Progress has been made in understanding the post-SCI pathophysiological changes (2). However, improving neurological functional repair after damage still remains a challenge. Local inflammatory response is a major factor that influences damage repair (1). The inflammatory response is regarded as a double-edged sword. On the one hand, it can eliminate pathogens and necrotic tissue debris, thus establishing the conditions for axon regeneration and tissue remodeling (3). On the other hand, excessive congregation of neurotoxic inflammatory factors and mediators will also aggravate tissue damage (4). Thus, the role of inflammatory response in SCI remains unclear.

SCI manifests as restricted movement and pain in the corresponding damaged areas, including sphincter of Oddi dysfunction, dystonia and pathological reflex (3). SCI is the primary cause of damaged neurons and neurogliocyte death, so it has been a key focus of medical research. In addition, SCI is associated with perpetual spinal cord dysfunction (3).

In previous research, macrophages have been demonstrated to exhibit diversity and plasticity in different damaged micro-environments, known as macrophage polarization (3). Furthermore, they can secrete numerous proinflammatory mediators, including tumor necrosis factor (TNF)- $\alpha$, interleukin (IL)-12, IL-23 and nitric oxide (3). Therefore, it has proinflammatory activity and aggravates tissue damage (5).

Toll-like receptor-4 (TLR4) is a pattern recognition receptor, which is closely related to congenital immunity (6). TLR4 is a membrane receptor with leucine-rich repeat. As is reported in previous study, injection of lipopolysaccharide in the spinal cord can upregulate TLR4 expression and activate the inflammatory response (6). Thus, SCI has resulted in nerve injury and functional defect, which reveals that TLR4 is distributed in the spinal cord (3). SCI can destroy the normal structure of the spinal cord (3). Meanwhile, it promotes extensive contact of extracellular matrix (ECM) with inflammatory cells. ECM contains numerous endogenous molecules, including laminin, collagen and proteoglycan (3). It was suggested in previous 
research that some endogenous substances will be produced after SCI, including necrotic tissues and oxygen radicals. These substances may stimulate microvascular endothelial cells (3). Furthermore, NF- $\kappa \mathrm{B}$ is activated in nerve cells and gliocytes for nuclear translocation (3). In addition, the activated $N F-\kappa B$ can elevate transcriptional activities of many inflammatory factor genes, including intercellular adhesion molecule 1, IL-1, IL-6 and TNF- $\alpha$ (3). There are binding sites in enhancers and promoters of inflammatory factors corresponding to those in NF- $\kappa \mathrm{B}$. In this way, the inflammatory response in damage regions is upregulated and the severity of tissue damage is increased (3). Zhong et al (7) reported that the TLR4/NF- $\kappa \mathrm{B} / \mathrm{miRNA}$ (miR)-146a pathway contributes to the assisted reproductive technology-correlated preterm birth outcome. In the present study, the neuroprotective effects of miR-146a in SCI, as well as the possible underlying mechanisms of these effects, were investigated.

\section{Materials and methods}

Animals and treatments. A total of 16 adult female Sprague-Dawley rats (200-220 g; 6-8 weeks) were purchased from the Animal Laboratory of Guangzhou Medical University (Guangzhou, China) and housed at $23 \pm 1^{\circ} \mathrm{C}$ with a humidity of $50-60 \%$, in a 12 -h light/dark cycle. The study protocol was approved by the Institutional Animal Care and Use Committee of Shandong University. All rats $(n=16)$ were randomized to a control group $(n=6)$ or an SCI model group $(n=10)$. All rats were anesthetized with intraperitoneal $300 \mathrm{mg} / \mathrm{kg}$ chloral hydrate (Sigma-Aldrich; Merck KGaA, Darmstadt, Germany) and the T8-T10 spinal column was exposed. The Impact One ${ }^{\mathrm{TM}}$ Stereotaxic Impactor (Leica Microsystems GmbH, Wetzlar, Germany) was inserted through the atlanto-occipital membrane at T9 and was an SCI model was established as previously described (8). Rats of the sham group were anesthetized with intraperitoneal $300 \mathrm{mg} / \mathrm{kg}$ chloral hydrate (Sigma-Aldrich; Merck KGaA) without the induction of SCI.

Reverse transcription-quantitative polymerase chain reaction (RT-qPCR). Total cellular RNA was isolated from spinal cord tissue or PC-12 cells transfected using the TRIzol (Invitrogen; Thermo Fisher Scientific, Inc., Waltham, MA, USA) and cDNA synthesis was performed using the TaqMan MicroRNA RT kit (Applied Biosystems; Thermo Fisher Scientific, Inc.). qPCR was performed using a Prism 7000 Real-Time PCR system with Power SYBR Green Master mix (Applied Biosystems; Thermo Fisher Scientific, Inc.). The conditions were as follows: $95^{\circ} \mathrm{C}$ for $5 \mathrm{~min}$, followed by 40 cycles of three-step PCR $\left(95^{\circ} \mathrm{C}\right.$ for $30 \mathrm{sec}, 60^{\circ} \mathrm{C}$ for $30 \mathrm{sec}$ and $72^{\circ} \mathrm{C}$ for $30 \mathrm{sec}$ ). The following primer sequences were utilized: miR-146a sense, $5^{\prime}$-GCGAGG TCAAGTCACTAGTGGT-3' and antisense, 5'-CGAGAAGCT TGCATCACCAGAGAACG-3'; U6 sense, 5'-CTCGCTTCG GCAGCACA-3' and antisense, 5'-AACGCTTCACGAATT TGCGT-3'. miRNA expression was calculated using the $2^{-\Delta \Delta C q}$ method (9).

Cell lines and cell culture. PC-12 cells were purchased from Shanghai library of Chinese academy of sciences (Shanghai, China) and cultured in Dulbecco's modified Eagle's medium

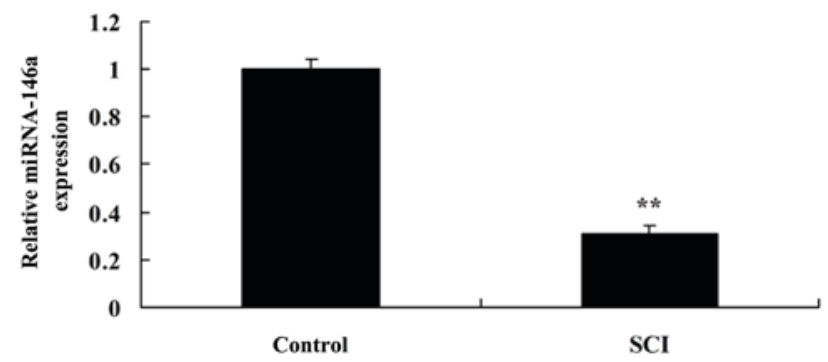

Figure 1. Expression of microRNA-146a in an SCI rat model. ${ }^{* *} \mathrm{P}<0.01$ vs. control. SCI, spinal cord injury.

(Invitrogen; Thermo Fisher Scientific, Inc.) supplemented with $10 \%$ fetal bovine serum (Gibco; Thermo Fisher Scientific, Inc.) at $37^{\circ} \mathrm{C}$ in a humidified atmosphere containing $5 \% \mathrm{CO}_{2} .100 \mathrm{ng}$ of miR-146a mimics (UGAGAACUGAAUUCCAUGGGUU), $100 \mathrm{ng}$ of anti-miR-146a mimics (AACCCAUGGAAUUCA GUUCUCA) and $100 \mathrm{ng}$ of negative control mimics (AAA AAAAAAA) were transfected into cells $\left(1 \times 10^{6}\right.$ cell $\left./ \mathrm{ml}\right)$ using Lipofectamine 2000 (Invitrogen; Thermo Fisher Scientific, Inc.), according to the manufacturer's protocol. After transfection for $48 \mathrm{~h}$, an SCI model was induced in PC-12 cells $\left(1 \times 10^{6} \mathrm{cell} / \mathrm{ml}\right)$ using $100 \mathrm{ng} / \mathrm{ml}$ of LPS for $4 \mathrm{~h}$ at $37^{\circ} \mathrm{C}$. Next, $2.5 \mathrm{nM}$ of TAK-242, a TLR4 inhibitor was added to the SCI in vitro model $\left(1 \times 10^{6}\right.$ cell $\left./ \mathrm{ml}\right)$ following anti-miR-146a for $48 \mathrm{~h}$ at $37^{\circ} \mathrm{C}$.

Measurement of inflammation factors. PC-12 cells were collected following the induction of LPS for $4 \mathrm{~h}$ by centrifugation at $1,000 \mathrm{x}$ g for $10 \mathrm{~min}$ at $4^{\circ} \mathrm{C}$. TNF- $\alpha$ (ab100747), IL- $1 \beta$ (ab100704) and IL-6 (ab100712) content was measured using ELISA kits (Abcam, Cambridge, MA, USA). Absorbance was measured at $450 \mathrm{~nm}$ using a microplate reader (model 550; Bio-Rad Laboratories, Inc., Hercules, CA, USA).

Western blot analysis. PC-12 cells $\left(2 \times 10^{6}\right.$ cell $\left./ \mathrm{ml}\right)$ were homogenized in radioimmunoprecipitation assay lysis buffer (Beyotime Institute of Biotechnology, Nanjing, China) on ice and protein was quantified using BCA buffer (Beyotime Institute of Biotechnology). Protein separation $(50 \mu \mathrm{g} / \mathrm{per}$ lane) was performed using 8-10\% SDS-PAGE, followed by transferring onto a polyvinylidene difluoride membrane. The membrane was blocked with $5 \%$ skim milk for $1 \mathrm{~h}$ at $37^{\circ} \mathrm{C}$, then incubated overnight at $4^{\circ} \mathrm{C}$ with primary antibodies: Anti-inducible nitric oxide synthase (iNOS; sc-649, 1:300), anti-prostaglandin E2 (PGE2; sc-20676, 1:500), anti-TLR4 (sc-10741, 1:500), anti-myeloid differentiation primary response 88 (MyD88; sc-11356, 1:500), anti-nuclear factor $\kappa \mathrm{B}$ (NF-кB; sc-109, 1:200) and anti-GADPH (sc-25778, 1:2,000) (all from Santa Cruz Biotechnology, Inc., Dallas, TX, USA). The membrane was then incubated with peroxidase anti-rabbit IgG (sc-2004, 1:5,000, Santa Cruz Biotechnology, Inc.) for $1 \mathrm{~h}$ at $37^{\circ} \mathrm{C}$. The results were detected using an enhanced chemiluminescence kit (Santa Cruz Biotechnology, Inc.) and analyzed using sodium Image Lab 3.0 (Bio-Rad Laboratories, Inc.).

Statistical analysis. Data are expressed as the mean \pm standard deviation using SPSS 17.0 (SPSS, Inc., Chicago, IL, USA). Differences between results were assessed using one-way 

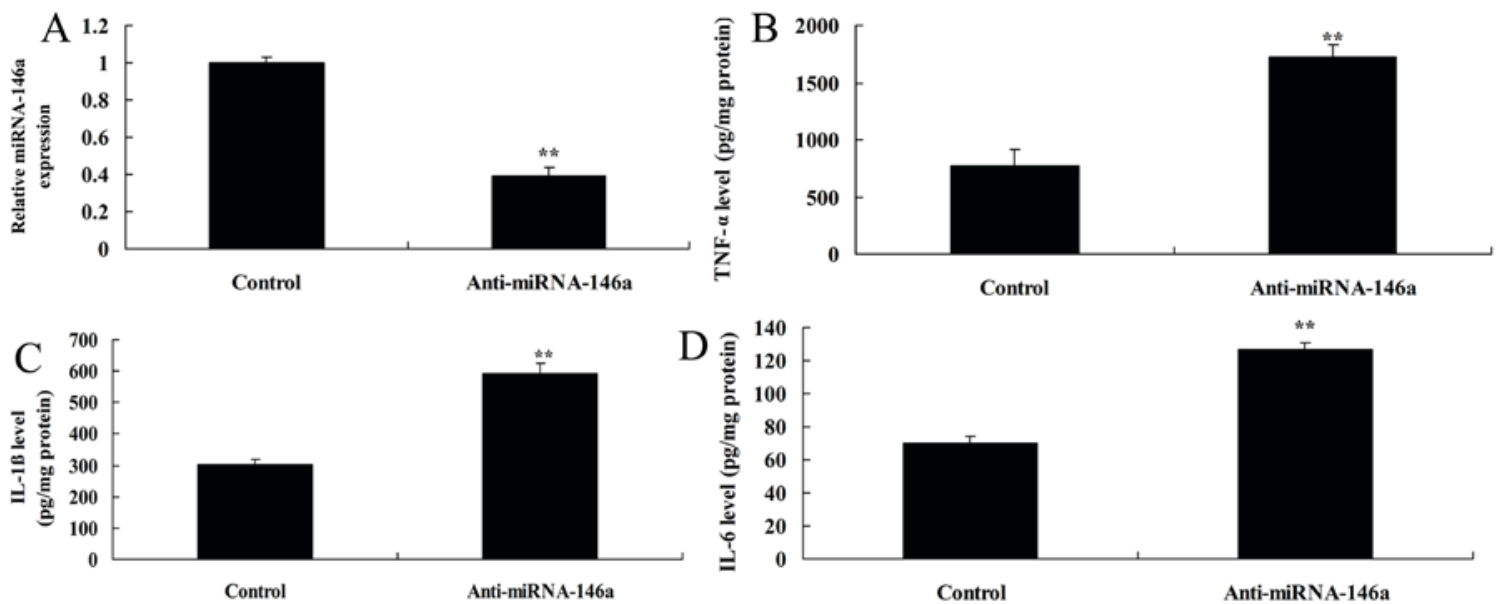

Figure 2. Downregulation of miRNA-146a increases inflammation in a spinal cord injury model in vitro. (A) miR-146a expression. (B) TNF- $\alpha$, (C) IL-1 $\beta$ and (D) IL-6 protein levels. ** $\mathrm{P}<0.01$ vs. control. Control, negative control group; anti-miRNA-146a, transfection with anti-miRNA-146a mimics; miRNA, microRNA, TNF, tumor necrosis factor; IL, interleukin.
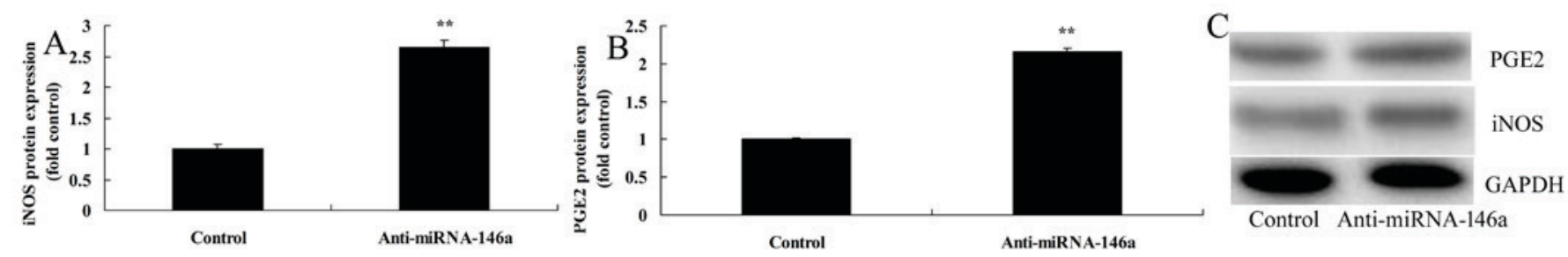

Figure 3. Downregulation of miRNA-146a enhances iNOS and PGE2 protein expression in a spinal cord injury model in vitro. iNOS and PGE2 protein expression were evaluated by western blot analysis. (A and B) Quantified analysis. (C) Representative western blots. ${ }^{* *} \mathrm{P}<0.01$ vs. control. Control, negative control group; anti-miRNA-146a, transfection with anti-miRNA-146a mimics; miRNA, microRNA; iNOS, inducible nitric oxide synthase; PGE2, prostaglandin E2.

analysis of variance followed by Tukey's post-hoc test. $\mathrm{P}<0.05$ was considered to indicate a statistically significant difference.

\section{Results}

Expression level of miR-146a in an SCI rat model. As indicated in Fig. 1, miR-146a expression was downregulated in the spinal cord tissue of the SCI rat model compared with the control group. These results suggested that miR-146a may be involved in the regulation of inflammation in SCI.

Downregulation of miR-146a increases inflammation in an SCI model in vitro. miR-146a expression was downregulated in an in vitro model of SCI using anti-miR-146a mimics. As indicated in Fig. 2A, there was significant inhibition of miR-146a expression in the in vitro model of SCI transfected with anti-miR-146a compared with the control group. Furthermore, TNF- $\alpha$, IL- $1 \beta$ and IL- 6 levels were significantly higher in the miR-146a downregulation group compared with the control group (Fig. 2B-D).

Downregulation of miR-146a enhances iNOS and PGE2 protein expression in an SCI model in vitro. PGE2 and iNOS expression were evaluated in an SCI model in vitro by western blot analysis. The results indicated that iNOS and PGE2 protein expression were significantly increased following miR-146a downregulation compared with the control group (Fig. 3).
Downregulation of miR-146a increases the level of TLR4, $M y D 88$ and $N F-\kappa B$ expression in an SCI model in vitro. In order to evaluate the mechanism of the anti-inflammatory effect of miR-146a on SCI, the TLR4/MyD88/NF-kB signaling pathway was examined in an SCI model following treatment with anti-miR-146a. As indicated in Fig. 4, inhibition of miR-146a significantly promoted TLR4, MyD88 and NF- $\mathrm{BB}$ protein expression compared with the control group.

Upregulation of miR-146a decreases inflammation in an SCI model in vitro. In order to evaluate whether upregulation of miR-146a would affect inflammation in an SCI model in vitro, cells were transfected with miR-146a mimics. As indicated in Fig. 5A, miR-146a expression was significantly upregulated in the SCI model transfected with miR-146a mimics compared with the control group. The level of expression of TNF- $\alpha$, IL-1 $\beta$ and IL- 6 was significantly lower in the group with miR-146a upregulation compared with the control group (Fig. 5B-D).

Upregulation of miR-146a inhibits iNOS and PGE2 protein expression in an SCI model in vitro. Upregulation of miR-146a significantly inhibited iNOS and PGE2 protein expression compared with the control group (Fig. 6).

Upregulation of miR-146a suppresses TLR4, MyD88 and $N F-\kappa B$ level expression in SCI model vitro. As expected, upregulation of miR-146a significantly suppressed the levels 

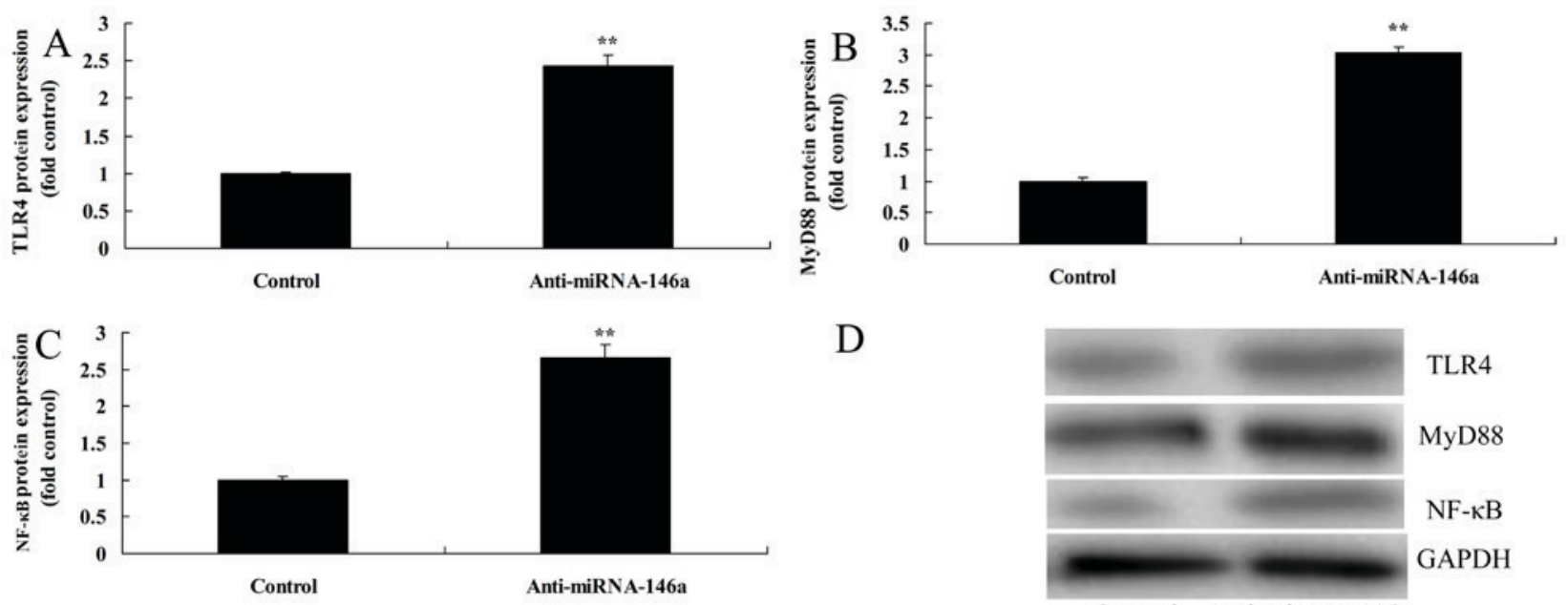

$\mathrm{D}$

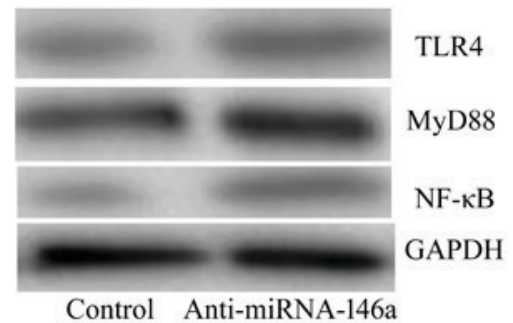

Figure 4. Downregulation of miRNA-146a increases TLR4, MyD88 and NF- $\mathrm{kB}$ expression levels in a spinal cord injury model in vitro. (A-C) Quantified analysis of TLR4, MyD88 and NF-kB protein expression. (D) Representative western blots. ${ }^{* *} \mathrm{P}<0.01$ vs. control. Control, negative control group; anti-miRNA-146a, transfection with anti-miRNA-146a mimics; miRNA, microRNA; TLR4, Toll-like receptor 4; MyD88, myeloid differentiation primary response 88; NF-кB, nuclear factor $\kappa \mathrm{B}$.
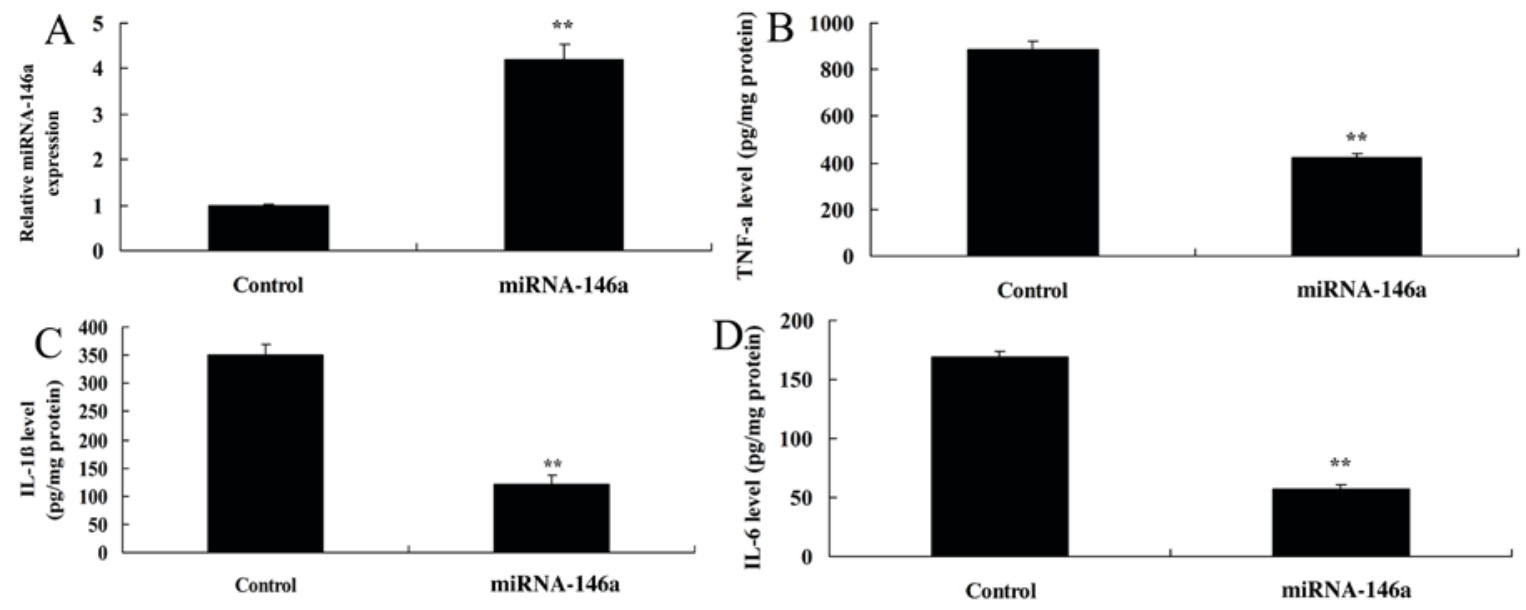

Figure 5. Upregulation of miRNA-146a decreases inflammation in a spinal cord injury model in vitro. (A) miRNA-146a expression. (B) TNF- $\alpha$, (C) IL-1 $\beta$ and (D) IL-6 protein levels. "** $\mathrm{P}<0.01$ vs. control. Control, negative control group; miRNA-146a, transfection with miRNA-146a mimics; miRNA, microRNA; TNF, tumor necrosis factor; IL, interleukin.

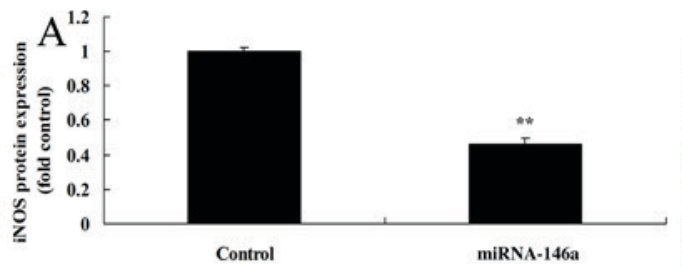

Figure 6. Upregulation of miRNA-146a inhibits iNOS and PGE2 protein expression in a spinal cord injury model in vitro. iNOS and PGE2 protein expression was evaluated by western blot analysis. (A and B) Quantified analysis. (C) Representative western blots. "* $\mathrm{P}<0.01$ vs. control. Control, negative control group; miRNA-146a, transfection with miRNA-146a mimics; miRNA, microRNA; iNOS, inducible nitric oxide synthase; PGE2, prostaglandin E2.

of TLR4, MyD88 and NF- $\kappa \mathrm{B}$ expression compared with the control group (Fig. 7).

Inhibition of TLR4 attenuates the proinflammatory effects of anti-miR-146a in an SCI model in vitro. In order to evaluate the function of TLR4 in the proinflammatory effects of
anti-miR-146a in SCI, $2.5 \mathrm{nM}$ of TAK-242, a TLR4 inhibitor, was administered following transfection with anti-miR-146a. As indicated in Fig. 8, inhibition of TLR4 significantly attenuated the proinflammatory effects of anti-miR-146a on TLR4, MyD88 and NF- $\mathrm{BB}$ expression compared with the anti-miR-146a group. In addition, inhibition of TLR4 

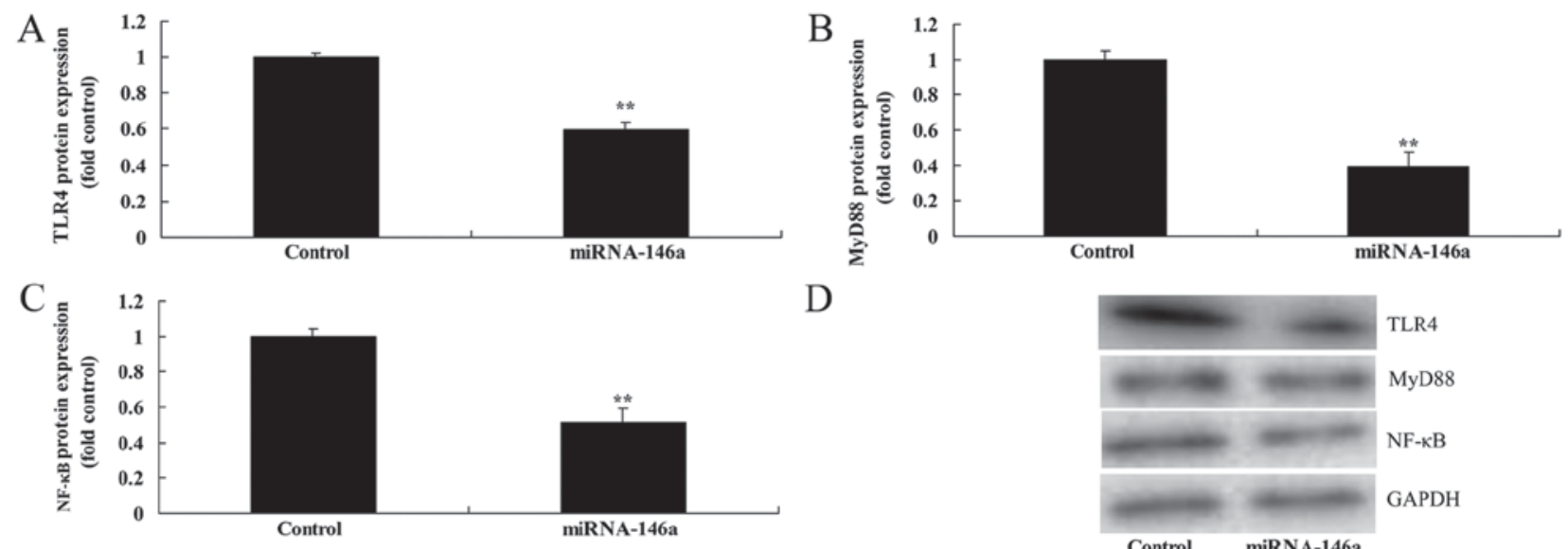

$\mathrm{D}$

Figure 7. Upregulation of miRNA-146a suppresses TLR4, MyD88 and NF- $\mathrm{kB}$ expression levels in a spinal cord injury model in vitro. TLR4, MyD88 and NF- $\mathrm{kB}$ protein expression was evaluated by western blot analysis. (A-C) Quantified analysis. (D) Representative western blots. "* $<<0.01$ vs. control. Control, negative control group; miRNA-146a, transfection with miRNA-146a mimics; miRNA, microRNA; TLR4, Toll-like receptor 4; MyD88, myeloid differentiation primary response 88 ; NF- $\kappa$ B, nuclear factor $\kappa \mathrm{B}$.
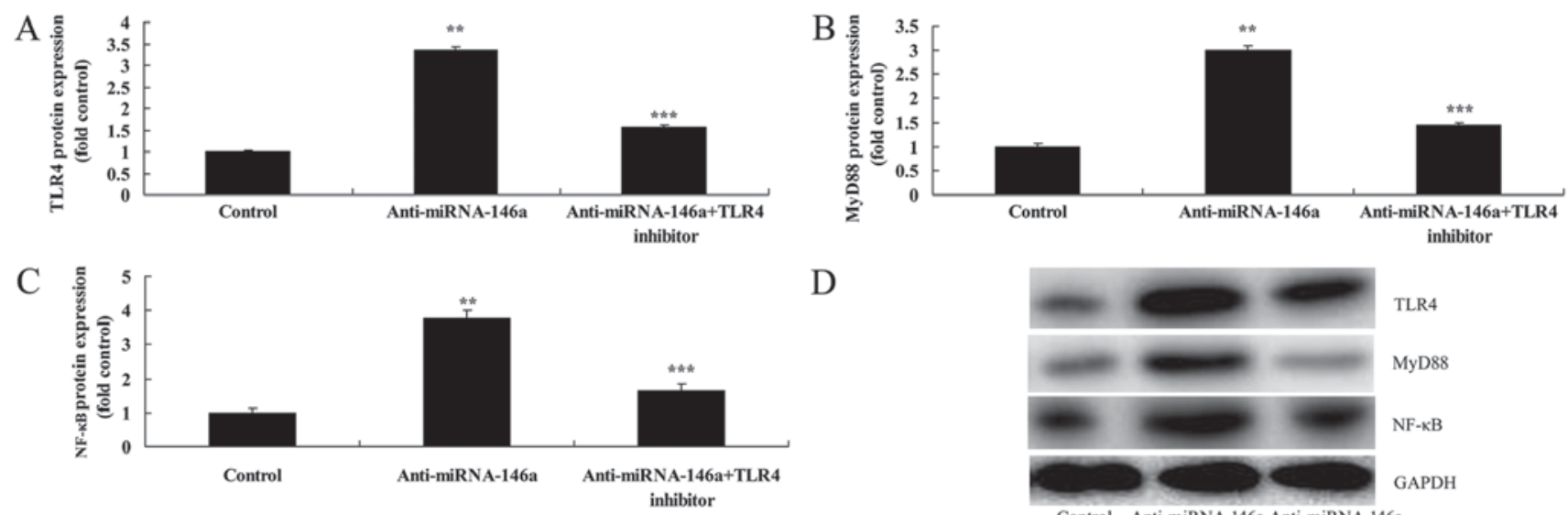

$\mathrm{D}$

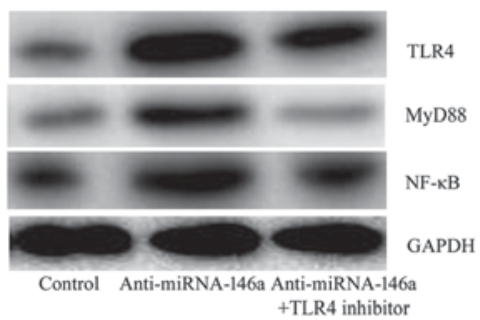

Figure 8. TLR4 inhibition attenuates the proinflammatory effects of anti-miRNA-146a on TLR4, MyD88 and NF-kB expression levels in a spinal cord injury model in vitro. TLR4, MyD88 and NF- $\mathrm{KB}$ protein expression was evaluated by western blot analysis. (A-C) Quantified analysis. (D) Representative western blots. ${ }^{* *} \mathrm{P}<0.01$ vs. control; ${ }^{* * *} \mathrm{P}<0.01$ vs. anti-miRNA-146a. Control, negative control group; anti-miRNA-146a, transfection with anti-miRNA-146a mimics; miRNA, microRNA; TLR4, Toll-like receptor 4; MyD88, myeloid differentiation primary response 88; NF- $\mathrm{B}$, nuclear factor $\kappa \mathrm{B}$.

significantly attenuated the proinflammatory effects of anti-miR-146a on TNF- $\alpha$, IL-1 $\beta$ and IL-6 levels compared with the anti-miR-146a group (Fig. 9). Furthermore, inhibition of TLR4 significantly attenuated the proinflammatory effects of anti-miR-146a on iNOS and PGE2 protein expression compared with the anti-miR-146a group (Fig. 10).

\section{Discussion}

SCI refers to direct or indirect external trauma to normal spine and spinal cord tissues, which impacts spinal cord functions (3). For instance, this may be caused by car accidents or falls. It was demonstrated in the current study that miR-146a expression is downregulated in an SCI rat model compared with the control group. Zhang et al (10) reported that miR-146a could inhibit the activities of inflammatory factors and NF- $\mathrm{\kappa B}$ in lupus nephritis. In the current study, only one SCI rat model was used, but other SCI animal models exist, including an LPS-induced SCI animal model of chronic cervical SCI (11).
This LPS-induced SCI animal model is a bacterial infection model, which simulates patients with menopause SCI or uterine trauma SCI; by contrast the current study induced damage at T9 for a classic SCI animal model. This immediately destroys spinal cord to generate an acute SCI model.

The phenotype of macrophage polarity can be altered under different inflammatory micro-environments (3). As key components of an inflammatory micro-environment, inflammatory factors also serve key functions in post-SCI nerve injury repair. This involves proinflammatory factors, including IL-1 $\beta$, TNF- $\alpha$, IL-12, IL- 6 and iNOS, as well as inflammatory cytokines, including IL-4, IL-10 and IL-13 (3). Results from a previous study indicated that overexpression of proinflammatory factors can promote apoptosis and further aggravate nerve injury (3). Compared with the wild type, the post-SCI damage area in IL-1 $\beta$ knockout mouse was markedly reduced. Furthermore, IL-1 $\beta$ can enhance neuroplasticity and promote recovery of motor function (3). TNF- $\alpha$ has cytotoxic effects on nerve cells and oligodendroglia cells (12). In addition, the TNF 

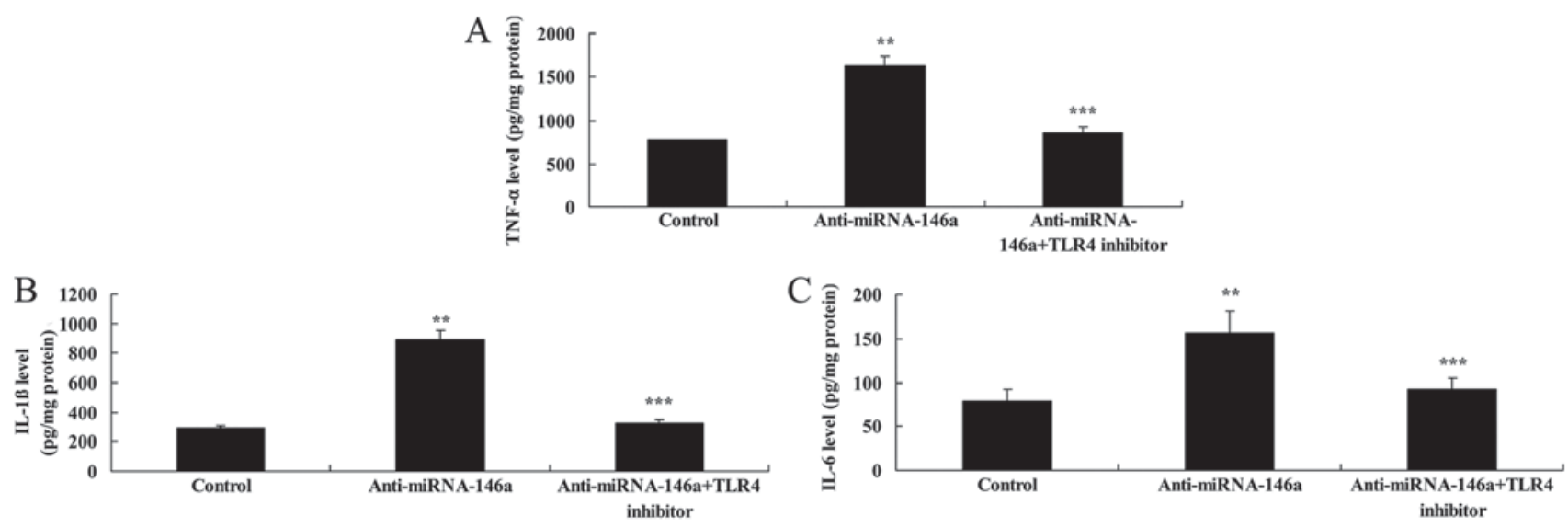

Figure 9. TLR4 inhibition attenuates the proinflammatory effects of anti-miRNA-146a on TNF- $\alpha$, IL-1 $\beta$ and IL-6 protein levels in a spinal cord injury model in vitro. (A) TNF- $\alpha$, (B) IL-1 $\beta$ and (C) IL-6 protein levels. ${ }^{* *} \mathrm{P}<0.01$ vs. control; ${ }^{* * *} \mathrm{P}<0.01$ vs. anti-miRNA-146a. Control, negative control group; anti-miRNA-146a, transfection with anti-miRNA-146a mimics; miRNA, microRNA; TNF, tumor necrosis factor; IL, interleukin; TLR4, Toll-like receptor 4.
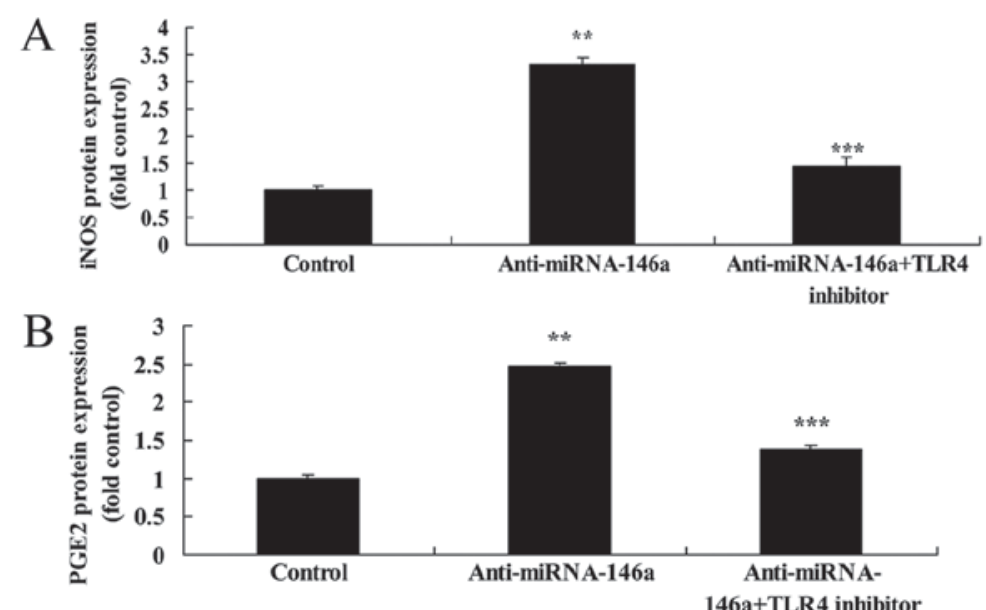

C

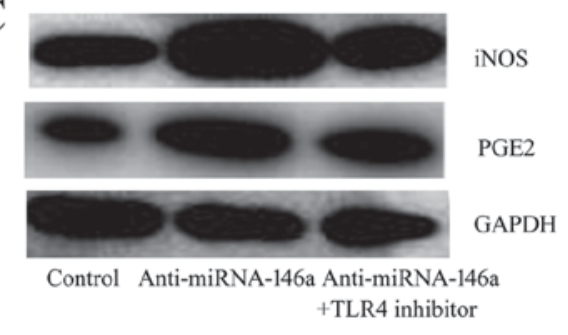

Figure 10. TLR4 inhibition attenuates the proinflammatory effects of anti-miRNA-146a on iNOS and PGE2 protein expression in a spinal cord injury model in vitro. iNOS and PGE2 protein expression was evaluated by western blot analysis. (A and B) Quantified analysis. (C) Representative western blots. ${ }^{* *} \mathrm{P}<0.01$ vs. control; ${ }^{* * *} \mathrm{P}<0.01$ vs. anti-miRNA-146a. Control, negative control group; anti-miRNA-146a, transfection with anti-miRNA-146a mimics; miRNA, microRNA; iNOS, inducible nitric oxide synthase; PGE2, prostaglandin E2; TLR4, Toll-like receptor 4.

superfamily receptor $1 \mathrm{~A}$ (TNFR1) can neutralize damage induced by TNF- $\alpha$ by preventing TNF- $\alpha$ receptor binding, so as to reduce TNF- $\alpha$ receptor binding on nerve cells (3). Applying TNFR1 in post-SCI local damage can notably reduce neuron death (3). By contrast, inflammatory cytokine IL-10 exerts important effects in regulating inflammatory responses, including anti-inflammation, apoptosis inhibition and neurotrophy (13). In the present study, upregulated miR-146a could decrease inflammation and inhibit expression of iNOS and PGE2 proteins in the SCI model in vitro.

In recent research, nerve cells were identified to be part of TLRs (3). It was originally thought that TLRs were primarily involved in immunity-related diseases. However, an increasing number of studies have suggested that TLRs are not only involved in the pathophysiological changes of neurodegenerative diseases, but also serve key functions in disease occurrence (3). Notably, the majority of TLRs exert their functions via the MyD88 pathway. Furthermore, TLR3 and TLR4 exert their functions in a MyD88 pathway-independent manner (3). Expression of these inflammatory factors may aggravate the secondary damage of spinal cord. The TLR4 signaling pathway can be classified into the MyD88-dependent and MyD88-independent pathways for cell transmission (3). TLR4 can activate IL-1 receptor-associated kinase through the MyD88-dependent pathway, thus further activating TNF receptor-associated factor 6 . Subsequently, it activates the downstream transcription factors $\mathrm{NF}-\kappa \mathrm{B}$, activator protein 1 and interferon regulatory factor 5 (3). These transcription factors can thus further induce expression of proinflammatory factors, including IL-6, TNF- $\alpha$ and IL-12 (3). As identified in the current study, upregulation of miR-146a can significantly suppress the expression levels of TLR4, MyD88 and NF- $\kappa$ B in an SCI model in vitro.

$\mathrm{NF}-\kappa \mathrm{B}$ is the generic term of the dimer transcription factor family. Every monomer of the dimer contains the Rel region, a sequence of 300 amino acids (3). The Rel region can bind with DNA as well as inhibitory molecules. Of these, the inhibitory molecule I $\kappa$ B contains 5-7 ankyrin repeat domains (3), with 30 amino acids in each domain. The domain is responsible for binding with the Rel region of $N F-\kappa B(3)$. NF- $\kappa B$ can 
be transferred into the nucleus following activation, so as to bind with relevant genes of multiple inflammatory factors and induce expression of proinflammatory factors (14). Activated TLRs can degrade $\mathrm{I} \kappa \mathrm{B}$ to activate $\mathrm{NF}-\kappa \mathrm{B}$, thus regulating the inflammatory response. Bao et al (14) reported that NF- $\kappa \mathrm{B}$ can be activated within $30 \mathrm{~min}$ of SCI. Thus, NF- $\kappa \mathrm{B}$ may also be involved in the pathophysiology of secondary damage after SCI. In addition, it was observed in the current study that TLR4 inhibition could attenuate the proinflammatory effects of anti-miR-146a in an SCI model in vitro. The present study suggests that miR-146a can suppress inflammation in SCI through the TLR4/MyD88/NF- $\mathrm{B}$ signaling pathway, which contributes to neural regeneration. The present study was limited by only evaluating miR-146a regulation of TLR4/MyD88/NF- $\kappa$ B signaling. Other inflammation signaling pathways, including $\mathrm{p}-\mathrm{NF}-\kappa \mathrm{B}$ and $\mathrm{p}-\mathrm{I} \kappa \mathrm{B}$ expression, will be investigated in future studies.

In summary, the present study demonstrated that miR-146a exerts an anti-inflammatory effect in SCI, which can be, at least in part, attributed to its modulation of the TLR4/MyD88/NF- $\mathrm{B}$ signaling pathway. These findings suggest that miR-146a may be a promising therapeutic agent for SCI.

\section{Competing interests}

The authors declare that they have no competing interests.

\section{References}

1. Ahmed MM, King KC, Pearce SM, Ramsey MA, Miranpuri GS and Resnick DK: Novel targets for spinal cord injury related neuropathic pain. Ann Neurosci 18: 162-167, 2011.

2. Hooshmand MJ, Galvan MD, Partida E and Anderson AJ: Characterization of recovery, repair, and inflammatory processes following contusion spinal cord injury in old female rats: Is age a limitation? Immun Ageing 11: 15, 2014.
3. Bank M, Stein A, Sison C, Glazer A, Jassal N, McCarthy D, Shatzer M, Hahn B, Chugh R, Davies P and Bloom O: Elevated circulating levels of the pro-inflammatory cytokine macrophage migration inhibitory factor in individuals with acute spinal cord injury. Arch Phys Med Rehabil 96: 633-644, 2015.

4. Kwan T, Floyd CL, Kim S and King PH: RNA binding protein $\mathrm{HuR}$ is Translocated in astrocytes following spinal cord injury and promotes the inflammatory response. J Neurotrauma 34 : 1249-1259, 2017

5. Jiang W, Li M, He F, Bian Z, Liu J, He Q, Wang X, Sun T and Zhu L: Dopamine D1 receptor agonist A-68930 inhibits NLRP3 inflammasome activation and protects rats from spinal cord injury-induced acute lung injury. Spinal Cord 54: 951-956, 2016.

6. Jia H, Xu S, Liu Q, Liu J, Xu J, Li W, Jin Y and Ji Q: Effect of pioglitazone on neuropathic pain and spinal expression of TLR-4 and cytokines. Exp Ther Med 12: 2644-2650, 2016.

7. Zheng CZ, Shu YB, Luo YL and Luo J: The role of miR-146a in modulating TRAF6-induced inflammation during lupus nephritis. Eur Rev Med Pharmacol Sci 21: 1041-1048, 2017.

8. Xin DQ, Hu ZM, Huo HJ, Yang XJ, Han D, Xing WH, Zhao Y and Qiu QH: Schisandrin B attenuates the inflammatory response, oxidative stress and apoptosis induced by traumatic spinal cord injury via inhibition of p53 signaling in adult rats. Mol Med Rep 16: 533-538, 2017.

9. Livak KJ and Schmittgen TD: Analysis of relative gene expression data using real-time quantitative PCR and the 2(-Delta Delta C(T)) method. Methods 25: 402-408, 2001.

10. Zhang K, Wu S, Li Z and Zhou J: MicroRNA-211/BDNF axis regulates LPS-induced proliferation of normal human astrocyte through PI3K/AKT pathway. Biosci Rep 37: pii: BSR20170755, 2017.

11. Suzuki H, Ahuja CS, Salewski RP, Li L, Satkunendrarajah K, Nagoshi N, Shibata S and Fehlings MG: Neural stem cell mediated recovery is enhanced by Chondroitinase $\mathrm{ABC}$ pretreatment in chronic cervical spinal cord injury. PLoS One 12: e0182339, 2017.

12. Pan W and Kastin AJ: Spinal cord injury changes cytokine transport. CNS Neurol Disord Drug Targets 15: 1139-1150, 2016.

13. Paterniti I, Campolo M, Cordaro M, Impellizzeri D, Siracusa R, Crupi R, Esposito E and Cuzzocrea S: PPAR- $\alpha$ modulates the Anti-inflammatory effect of melatonin in the secondary events of spinal cord injury. Mol Neurobiol 54: 5973-5987, 2017.

14. Bao G, Li C, Qi L, Wang N and He B: Tetrandrine protects against oxygen-glucose-serum deprivation/reoxygenation-induced injury via PI3K/AKT/NF- $\mathrm{KB}$ signaling pathway in rat spinal cord astrocytes. Biomed Pharmacother 84: 925-930, 2016. 